\title{
A Study on the Clinical Application of a New Type of Integrated Suctioning Semi-Rigid Ureteroscopic Lithotripsy for Ureteral Calculi
}

\section{Difu Fan ( $\square$ fandifu@126.com )}

The Affiliated Ganzhou Hospital of Nanchang University, Ganzhou People's Hospital

\section{Xiaohui Liao}

Gan County District Dermatology Institute

Chuanxiang Luo

Liaobu Township Community Health Service Center

\section{Leming Song}

The Affiliated Ganzhou Hospital of Nanchang University, Ganzhou People's Hospital Jack Michel

Larkin Community Hospital

Donghua Xie

Xie Urology LLC

\section{Research Article}

Keywords: new type, integrated suctioning ureteroscopy, lithotripsy, clinical application

Posted Date: February 4th, 2022

DOI: https://doi.org/10.21203/rs.3.rs-1257637/v1

License: (c) (1) This work is licensed under a Creative Commons Attribution 4.0 International License.

Read Full License 


\section{Abstract}

OBJECTIVE: To explore the effect and clinical value of a new type of integrated suctioning semi-rigid ureteroscopic lithotripsy for treating ureteral calculi.

METHODS: Retrospective analysis the clinic data of 180 patients who underwent surgery for ureteral calculi from May 2019 to October 2020 in our department, which was divided into three groups, 60 cases each. Group A used traditional semi-rigid ureteroscopic lithotripsy, group B used a suctioning semi-rigid ureteroscopic lithotripsy with a sheath being connected to a vacuum device; group $C$ used a new type of suctioning integrated rigid ureteroscopic lithotripsy with a novelly designed ureteroscope.

RESULTS: One hundred and sixty-four cases of ureteroscopic lithotripsy were completed in one stage, and the remaining 16 cases were converted to other surgical methods due to difficulty in placing a suctioning sheath or entering the ureteroscope due to ureteral stricture. Compared with group $\mathrm{A}$, group $\mathrm{C}$ had a higher rate of stone stone-clearance rate at 30 days after surgery, a shorter operation time and fewer hospitalization days $(P<0.05)$; Compared with group $B$, group $C$ had a had a higher one-stage operation success rate, a shorter operation time and fewer hospitalization days $(P<0.05)$.

CONCLUSIONS: Compared with the other two types, the new type suctioning integrated semi-rigid ureteroscopic lithotripsy has certain advantages including reduced operation time and length of hospital stay, and higher level of minimally invasiveness for upper urinary calculi.

\section{Introduction}

At present, urinary calculi are common and frequently-occurring diseases in urology ${ }^{[1]}$, and minimally invasive ureteroscopic lithotripsy has been widely used in clinical practice ${ }^{[2]}$. In traditional ureteroscopy, because the water injection and drainage system does not form a continuous water circulation at the front end of the ureteroscopic lens, the gravel dust affects the observation field, and more complications will occur during the perioperative period ${ }^{[3]}$, such as: ureteral injury, low lithotripsy efficacy, easiness of stone migration to the kidneys, high intrarenal pelvic pressure, etc. ${ }^{[4]}$. We therefore designed a new type of integrated semi-rigid ureteroscope (Patent\# ZL 20192 0339071.2) for lithotripsy, including an independent water injection port and negative pressure suction channel, and compared with the traditional semi-rigid ureteroscopic lithotripsy and negative pressure suctioning semi-rigid ureteroscopic lithotripsy using a ureteral access sheath. There is no need for a ureteral access sheath using the new type ureteroscopic lithotripsy with several other advantages in the treatment of ureteral calculi and has achieved good clinical results. The report is as follows.

\section{Materials And Methods}

\section{General information}


From May 2019 to October 2020, 180 patients underwent surgery for ureteral calculus in our hospital. The patients of retrospective analysis were divided into three groups, 60 cases of each group, and the same surgeon performed surgery for all the patients. Group A used traditional semi-rigid ureteroscopic lithotripsy (Figure 1.1/Figure1.2), group B used negative pressure suctioning semi-rigid ureteroscopic lithotripsy with the aid of a ureteral access sheath (Figure 2.1/Figure2.2), and group $C$ used the new type negative pressure suctioning integrated semi-rigid ureteroscopic lithotripsy (Figure 3.1/Figure3.2). Among Group A patients, there were 32 males and 28 females with a body mass index (BMI) $(26.17 \pm 4.08) \mathrm{kg} / \mathrm{m}^{2}$; in Group B, there were 34 males and 26 females with a BMI $(25.03 \pm 3.37) \mathrm{kg} / \mathrm{m}^{2}$; in Group C, there were 35 males and $25 f$ emales with a BMI $(24.88 \pm 3.10) \mathrm{kg} / \mathrm{m}^{2}$. There was no significant difference between the three groups in terms of age, gender composition, body mass index, stone load and comorbidities $(P>0.05)($ Table 1,2). Inclusion criteria: $\otimes$ All patients underwent surgery for ureteral calculus in our

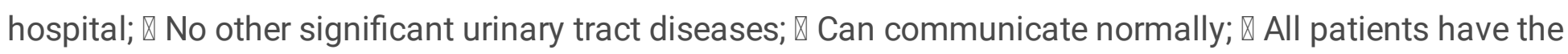
right to know, agree and sign the informed consent. Exclusion criteria: $\triangle$ accompanied by systemic hemorrhagic diseases; $₫$ suffering from stroke, coronary heart disease, and severe underlying diseases; $\mathbb{X}$ suffering from mental disorders; $₫$ poor compliance; $₫$ patients who withdrew from the study halfway. 
Table 1

Preoperative and perioperative data between Group A and Group C

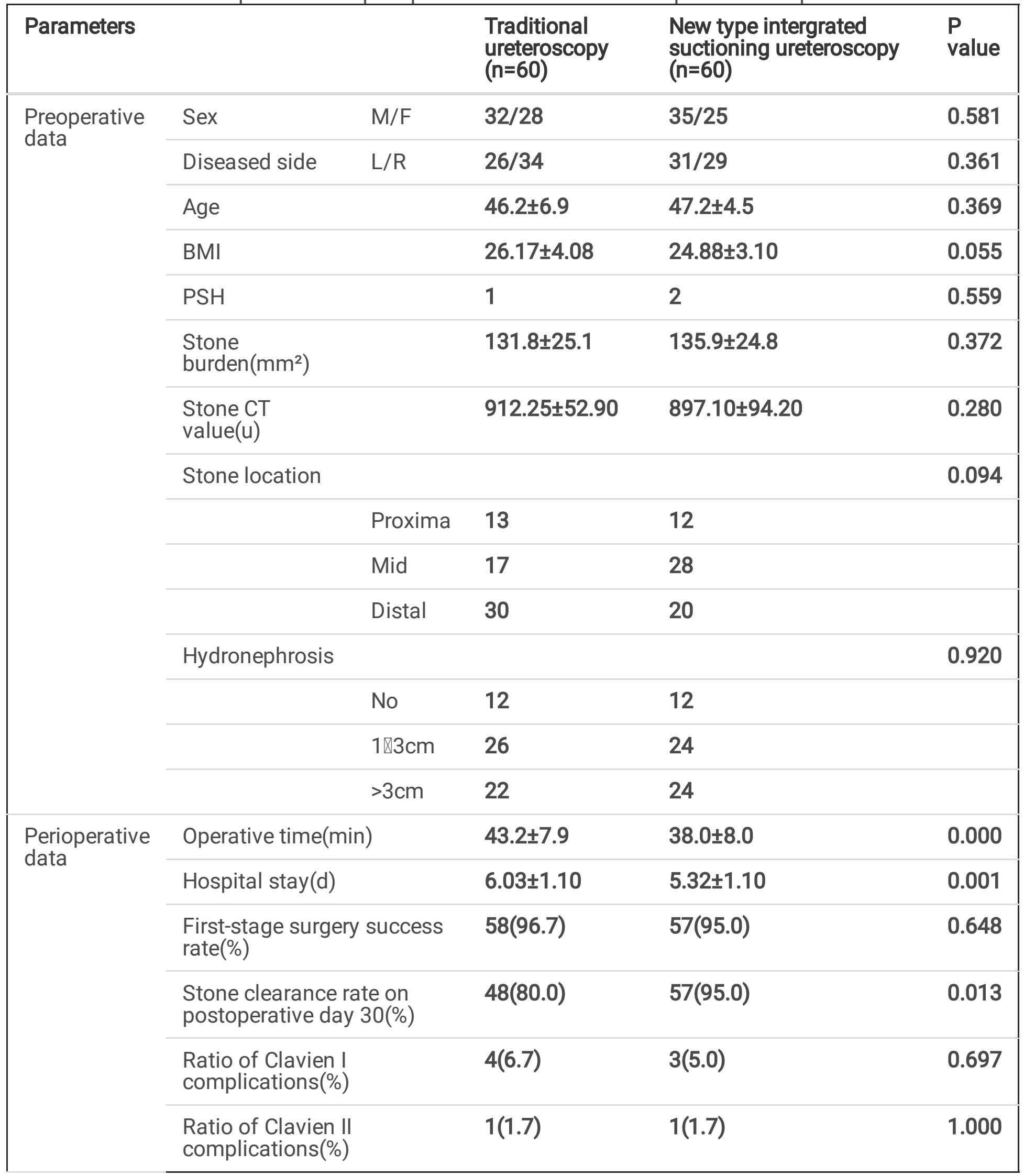


Table 2

Preoperative and perioperative data between Group B and Group C

\begin{tabular}{|c|c|c|c|c|c|}
\hline \multicolumn{3}{|l|}{ Parameters } & $\begin{array}{l}\text { Suctioning } \\
\text { ureteroscopy } \\
\text { with sheath } \\
(n=60)\end{array}$ & $\begin{array}{l}\text { New type intergraded } \\
\text { suctioning ureteroscopy } \\
(n=60)\end{array}$ & $\begin{array}{l}P \\
\text { value }\end{array}$ \\
\hline \multirow{15}{*}{$\begin{array}{l}\text { Preoperative } \\
\text { data }\end{array}$} & Sex & $\mathrm{M} / \mathrm{F}$ & $34 / 26$ & $35 / 25$ & 0.853 \\
\hline & Diseased side & $L / R$ & $23 / 37$ & $31 / 29$ & 0.142 \\
\hline & Age & & $45.7 \pm 6.5$ & $47.2 \pm 4.5$ & 0.149 \\
\hline & BMI & & $25.03 \pm 3.37$ & $24.88 \pm 3.10$ & 0.800 \\
\hline & $\mathrm{PSH}$ & & 1 & 2 & 0.559 \\
\hline & $\begin{array}{l}\text { Stone } \\
\text { burden }\left(\mathrm{mm}^{2}\right)\end{array}$ & & $136.5 \pm 26.6$ & $135.9 \pm 24.8$ & 0.887 \\
\hline & $\begin{array}{l}\text { Stone } C T \\
\text { value }(\mathrm{u})\end{array}$ & & $906.48 \pm 63.47$ & $897.10 \pm 94.20$ & 0.524 \\
\hline & Stone location & & & & 0.124 \\
\hline & & Proxima & 12 & 12 & \\
\hline & & Mid & 18 & 28 & \\
\hline & & Distal & 30 & 20 & \\
\hline & Hydronephrosis & & & & 0.750 \\
\hline & & No & 10 & 12 & \\
\hline & & $1 \otimes 3 \mathrm{~cm}$ & 28 & 24 & \\
\hline & & $>3 \mathrm{~cm}$ & 22 & 24 & \\
\hline \multirow{6}{*}{$\begin{array}{l}\text { Perioperative } \\
\text { data }\end{array}$} & \multicolumn{2}{|c|}{ Operative time(min) } & $41.6 \pm 10.6$ & $38.0 \pm 8.0$ & 0.035 \\
\hline & \multicolumn{2}{|l|}{ Hospital stay(d) } & $5.97 \pm 1.22$ & $5.32 \pm 1.10$ & 0.004 \\
\hline & \multicolumn{2}{|c|}{$\begin{array}{l}\text { First-stage surgery success } \\
\text { rate(\%) }\end{array}$} & 49(81.7) & $57(95.0)$ & 0.023 \\
\hline & \multicolumn{2}{|c|}{$\begin{array}{l}\text { Stone clearance rate on } \\
\text { postoperative day } 30(\%)\end{array}$} & $58(96.7)$ & $57(95.0)$ & 0.648 \\
\hline & \multicolumn{2}{|c|}{$\begin{array}{l}\text { Ratio of Clavien I } \\
\text { complications(\%) }\end{array}$} & $3(5.0)$ & $3(5.0)$ & 1.000 \\
\hline & \multicolumn{2}{|c|}{$\begin{array}{l}\text { Ratio of Clavien II } \\
\text { complications(\%) }\end{array}$} & $1(1.7)$ & $1(1.7)$ & 1.000 \\
\hline
\end{tabular}




\section{Methods}

All the patients were diagnosed with ureteral calculus by B-ultrasound, $\mathrm{KUB}+\mathrm{IVU}$, and CT with indications for surgery, and no absolute contraindications to surgery. Before the operation, the three groups of patients were routinely tested with blood and urine, and the mid-stage urine was collected for bacterial culture with drug sensitivity test. Preoperative urinary tract infections, renal insufficiency, hypertension and lung dysfunction were given symptomatic treatment to optimize the patient's condition, and surgery was performed after all parameters were improved. The surgical patients were then divided into three groups (groups A, B, and C), and each group underwent different transurethral ureteroscopic procedure.

Group A: Traditional semi-rigid ureteroscopic lithotripsy surgery group. Operation steps: After general anesthesia or combined lumbar and epidural anesthesia, patient was placed in lithotomy position and routine sterilization preparation was performed. An 8-9.8 Fr Wolf (Germany) single-channel semi-rigid ureteroscope was introduced into the bladder cavity. Following the zebra guide wire to the ureteral orifice, the scope was further advanced until the ureteral calculus was found. Holmium laser power parameter was adjusted to $0.6-1.0 \mathrm{~J} / 20-30 \mathrm{~Hz}(275 \mu \mathrm{m}$ fiber) for lithotripsy. Larger pieces of gravel can be removed from the body using stone-clamping clamps. After lithotripsy, a $5 \mathrm{Fr}$ double $\mathrm{J}$ tube was indwelled through the guide wire and a Foley catheter was indwelled.

Group B: negative pressure suctioning semi-rigid ureteroscopic lithotripsy with the aid of a ureteral access sheath UAS (a disposable minimally invasive expansion drainage kit from Shenzhen Kang Yi Bo Technology Development Co.,Ltd, China, medical device production license No.: 20122279) being connected to a vacuum suctioning device. Operation steps: Under general anesthesia or combined lumbar and epidural anesthesia and in a lithotomy position, following the insertion of zebra guide wire to the ureteral orifice until renal pelvis, a 12-14 Fr ureteral access sheath was then inserted over the guidewire, followed by a 6.8-7.5 Fr Wolf (Germany) single-channel semi-rigid ureteroscope. After finding the stones, the holmium laser power was adjusted to $0.6 \varangle 1.0 \mathrm{~J} / 20 \varangle 30 \mathrm{~Hz}$, and a $275 \mu \mathrm{m}$ optical holmium laser fiber was used to crush the stone. The small crushed stone powder was attracted with the water flow by vacuum suctioning to the stone collection bottle through the gap between the scope body and the ureteral access sheath; the slightly larger gravel debris will be attracted to the stone collection bottle with negative pressure suctioning by withdrawing the scope. After the lithotripsy was completed, a $5 \mathrm{Fr}$ double $\mathrm{J}$ stent was indwelled through the guide wire and a Foley catheter was indwelled. For those whom the ureteral access sheath was not successfully indwelled, middle and lower ureteral calculi were removed by holmium laser lithotripsy by inserting the ureteroscope directing with the guidance of the zebra guidewire, while upper ureteral calculi were removed by either percutaneous nephrolithotomy or flexible ureteroscopic lithotripsy based on patient's choice.

Group C: New type of negative pressure suctioning ureteroscopic lithotripsy surgery group. Operation steps: Under general anesthesia or combined lumbar and epidural anesthesia and in a lithotomy position, the $2 \mathrm{Fr}$ channel of a 7.5Fr Suode (Hangzhou, China) dual-lumen semi-rigid ureteroscope was connected to a three-way connector, the remaining two interfaces were connected to the water injection port and 
holmium laser fiber entrance. We then inserted a zebra guidewire through the $3 \mathrm{Fr}$ instrument channel of the ureteroscope. The scope was then advanced to the bladder cavity. Following the guidewire, the ureteroscope was inserted over the ureteral orifice and was further advanced until the ureteral calculus was found. The zebra guide wire was then removed and the ureteroscope was connected to vacuum suction tube. The holmium laser power parameter was then adjusted to $0.6-1.0 \mathrm{~J} / 20-30 \mathrm{~Hz}(275 \mu \mathrm{m}$ fiber). Lithotripsy was carried out in a "worm-eaten" pulverizing fashion. After crushing the stone, a $5 \mathrm{Fr}$ double $\mathrm{J}$ stent was then placed, and a Foley catheter was indwelled.

\section{Observation indicators}

$\nabla$ the basic data of the three groups of patients (gender, age, body mass index, stone size, calculus site, preoperative renal pelvis effusion); $\Downarrow$ operation time, hospitalization days, first-stage operation success rate, postoperative day 30 stone clearance rate; $₫$ Complications by Clavien system grading.

\section{Statistical methods}

In this study, SPSS20.0 software was used for statistical analysis of the research data. Measurement data was expressed as mean \pm standard deviation $(\Delta x \pm s)$, comparison between groups was performed by one-way analysis of variance (ANOVA), pairwise comparison was performed by pairwise comparison test due to different sample sizes in each group (Scheffe). Count data was expressed in terms of constituent ratio or rate (\%), and both group and pairwise comparisons were tested; $P<0.05$ is considered statistically significant.

\section{Results}

In 164 patients the surgery was completed by one stage. In the remaining 16 cases the surgeries were converted to other surgical methods due to the narrow ureter without being able to place a ureteral access sheath or advance the ureteroscope. Among the 16 cases, 10 patients with upper ureteral calculi were converted to minimally invasive percutaneous nephrolithotomy. 5 patients with upper ureteral calculi were cleared of the stone successfully by flexible ureteroscopic lithotripsy after indwelling a D-J stent for 4 weeks. One patient with a lower ureteral calculus was cleared of the stone successfully by semi-rigid ureteroscopic lithotripsy after indwelling a D-J stent for 4 weeks. There was no statistically significant difference in preoperative data among the three groups $(P>0.05)$. Among them, there were 2 cases in group $A, 11$ cases in group $B$, and 3 cases in group $C$ being converted to other surgical methods. Compared with group $A$, group $C$ had a higher rate of stone stone-clearance rate at 30 days after surgery, a shorter operation time and fewer hospitalization days $(P<0.05)$; Compared with group $B$, group $C$ had a had a higher one-stage operation success rate, a shorter operation time and fewer hospitalization days $(\mathrm{P}<0.05)$. There was low incidence of Clavien I and II complications in each group without statistical differences $(P>0.05)$. All these complications were cured after symptomatic treatment. There was no occurrence of Clavien system class III to V complications (Table 1,2).

\section{Discussion}


Ureteral stones are the most common upper urinary tract stones, the main symptoms are renal colic and hematuria, and the common complications are obstruction-induced pelvic effusion, infection, and renal function damage ${ }^{[5]}$. The current minimally invasive treatment methods include: extracorporeal shock wave lithotripsy (ESWL), semi-rigid ureteroscopic lithotripsy (URL), flexible ureteroscopic lithotripsy (RIRS), percutaneous nephrolithotomy (PCNL), laparoscopic ureterolithotomy (RPLU), etc. ${ }^{[6]}$, in which ureteroscopic lithotripsy is one of the most commonly used treatments ${ }^{[7]}$. Due to the impact of holmium laser lithotripsy dust on the observation field of traditional ureteroscopic lithotripsy ${ }^{[8]}$, it is easy to cause complications such as ureteral injury, reduced lithotripsy efficiency, stone migration to the kidney, and retrograde infection of the renal pelvis. Therefore, how to further improve the surgical safety and the efficiency of gravel clearance has become an urgent problem to be solved ${ }^{[9,10]}$. We have researched and designed a new type of integrated semi-rigid ureteroscopy with the aid of a three-way connector, which contains two independent perfusing and suctioning channels. The perfusing channel is also designed to accommodate the laser fiber, while the negative pressure suctioning channel runs through the entire scope body. During the operation, the negative pressure suction can immediately remove the gravel dust that affects the clarity of the visual field, and significantly improves the efficiency of stone graveling.

In the new type of integrated suctioning semi-rigid ureteroscopic lithotripsy operation, the perfusion flow rate is usually set to $50-60 \mathrm{ml} / \mathrm{min}$, the perfusion pressure is usually set to $150-200 \mathrm{mmHg}$, and the suction pressure is usually between 0.01-0.02 MPa. Affected by the condition of the ureter, the gap size between the scope and the inner wall of the ureter, and the size of the gravel, etc., the intraoperative parameters can be comprehensively adjusted according to the conditions of intraoperative ureteral filling and negative pressure suction patency, etc., to maintain the renal pelvis pressure within safe limits. As the ureteroscope is getting smaller and smaller, the clarity of the surgical field of vision is related to the pixels of the camera system, and the perfusion flow rate at the front of the scope becomes the most critical factor ${ }^{[8]}$. The new integrated semi-rigid ureteroscope contains two independent perfusion and suction channels, which can form a rapid continuous water circulation circuit at the front end of the ureteroscope. Intraoperatively, the continuous perfusion fluid at the front end of the ureteroscope can immediately remove the gravel dust of the holmium laser lithotripsy ${ }^{[11]}$, The surgical field of vision is clear, which significantly improves the efficiency of lithotripsy. Compared with group A and group B, the operation time and hospitalization time were significantly shorter in group $\mathrm{C}$ with several factors may account for. In this new type of ureteroscopy, a ureteral access sheath is not needed. The traditional ureteroscope entry method is not changed. Performance of every step under visual guidance can reduce the damage of the ureteral mucosa, promoting the rapid postoperative recovery of patients. This is consistent with the medical reform policy of vigorously promoting day surgery to accelerate the turnover of hospital beds in tertiary hospitals.

Due to the continuous water circulation that can be formed in the front of the ureteroscope during operation, the pressure gradient between the proximal and distal stone ends is small ${ }^{[12]}$, the impact force on the stones is therefore small and the stones are not easy to move and escape to the kidneys causing postoperative residual stones ${ }^{[13]}$. During the operation, $275 \mu \mathrm{m}$ holmium laser fiber was used to pulverize 
the stone in situ in a "worm eaten" fashion. The gravel particles were small and easily excreted with the urine. The double $\mathrm{J}$ stent was removed 30 days after the operation.

Suctioning ureteroscopic lithotripsy with the aid of a ureteral access sheath is the first choice for complex and heavy-duty ureteral calculi with feasible ureteral conditions ${ }^{[14,15,16]}$. However, there were approximately $15 \%$ of patients could not undergo one-stage ureteroscopic lithotripsy due to difficulty in placing the sheath because of ureteral distortion and stenosis ${ }^{[17,18]}$.

Our new type of negative pressure suctioning integrated semirigid ureteroscopy with the help of the novelly designed scope with two independent channels. The success rate of the first stage ureteroscopy is significantly improved, especially in patients with solitary kidney, narrow ureter that cannot be sheathed, and upper ureteral calculus with small stone load.

The study was performed by the same skilled urologist. There was no statistically significant difference in the incidence of complications in the total Clavien grade of patients in groups $A, B$, and C. It is revealed that the urologists participating in the study can master transurethral ureteroscopy lithotripsy and control the safety of the three types of ureteroscopic lithotripsy, and avoid ureteral perforation, false passage formation, avulsion and other surgical complications. The surgeon can control the balance of the amount of perfusion fluid ${ }^{[19]}$, maintain the internal pressure of the renal pelvis within a safe range, and avoid the occurrence of urinary sepsis that endangers the patient ${ }^{[20,21]}$.

In addition, the continuous perfusion flow in the new dual-channel integrated ureteroscope surgery is high, and the holmium laser heat is quickly dissipated, which can avoid the long-term complications of high temperature burns to the ureteral mucosa leading to ureteral stenosis ${ }^{[22]}$. There is no need to introduce the sheath of the ureteroscope during the operation, and the way of entering the ureteroscope is not changed, which reduces the risk of surgical operation, makes the operation more convenient, and promotes the postoperative recovery of the patient.

The main problems that should be paid attention to in the new type of negative pressure suctioning integrated rigid endoscope lithotripsy are: $₫$ During the operation, closely observe the changes in the clarity of the visual field and the flowing waste fluid in the negative pressure suction tube, and intermittently withdraw the scope to the bladder according to the situation during the operation, which is also conducive to the excretion of $1-2 \mathrm{~mm}$ gravel in the ureteral cavity into the bladder cavity with the

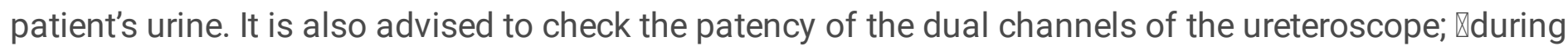
holmium laser lithotripsy, it should be performed in in situ as much as possible and in a fashion of "worm-eaten" to pulverize the stone, to prevent the gravel from blocking the suction channel, causing continuous perfusion and leading to renal pelvic hypertension. When the ureteroscope is intermittently retracted into the bladder cavity, the negative pressure suction interface is replaced with a water injection tube, which can judge and ensure the patency of the suctioning channel according to the clarity of the visual field. 
In summary, the three techniques of minimally invasive treatment of ureteral calculi with semi-rigid ureteroscopic lithotripsy are safe and effective. Compared with the other two surgical methods, the new type negative pressure suctioning integrated ureteroscopic lithotripsy has certain advantages. It can ensure a relatively high first-stage success rate of ureteroscopic entrance to reach the stone. Without using a disposable ureteral sheath, it can increase the drainage during the operation, significantly improve the clarity of the lithotripsy visual field during the operation, improve the lithotripsy efficiency, and significantly reduce the patient's hospital stay and expenses.

Nevertheless, there are still shortcomings in this study. The sample size of the study is relatively small, and it is a retrospective analysis. Therefore, the conclusions of this study need to be further confirmed by more in-depth research.

\section{Declarations}

Consent for publication: Written informed consent was obtained from the patients for publication of this report and any accompanying images. Copies of the written consent are available for review by the Editor of the journal.

Ethics approval and consent to participate: Ethical and regulatory approvals were sought and obtained from the Affiliated Ganzhou Hospital of Nanchang University. This trial will be performed in accordance with the World Medical Association Declaration of Helsinki. The study protocol has been approved by The Clinic Scientific Research Ethic Committee of Ganzhou People's Hospital, Ganzhou, China, (TY-ZKY2021001-01). The findings of the study will be published in peer-reviewed journals and presented at national and international scientific meetings. Written informed consent was obtained from all participants.

Competing Interests: None.

Acknowledgements: None.

Funding: This study was supported by Jiangxi Provincial Department of Science and Technology (Grant No: 20203BBGL73169).

Availability of data and materials: The datasets used and analyzed during the current study available from the corresponding author on reasonable request. The useful partial data generated or analyzed during this study are fully included in the supplementary information file. More details of the data cannot be mentioned due to patent issues.

\section{Authors' Contributions:}

Protocol/project development: Fan, Liao, Xie;

Data collection or management: Fan, Xie;

Data analysis: Fan, Luo, Xie; 
Manuscript writing/editing: Fan, Xie, Michel, Song.

\section{References}

1. Wang W, Fan J, Huang G, Li J, Zhu X, Tian Y, Su L. Prevalence of kidney stones in mainland China: A systematic review. Sci Rep. 2017;7:41630

2. Hou CP, Lin YH, Juang HH, Chang PL, Chen CL, Yang PS, Lee CC, Tsui KH. Effect of ureteral calculus in outpatients receiving semirigid ureteroscope laser lithotripsy. Medicine (Baltimore). 2020;99:e19324.

3. Taguchi K, Cho SY, Ng AC, Usawachintachit M, Tan YK, Deng YL, Shen CH, Gyawali P, Alenezi H, Basiri A, Bou S, Djojodemedjo T, Sarica K, Shi L, Singam P, Singh SK, Yasui T. The Urological Association of Asia clinical guideline for urinary stone disease. Int J Urol. 2019;26:688-709

4. Zhong W, Leto G, Wang $L$, et al. Systemic inflammatory response syndrome after flexible ureteroscopic lithotripsy: a study of risk factors. Journal of Endourology, 2014, 29:25-28.

5. Palle J S Osther, Katja V Pedersen, Søren K Lildal, Maria S Pless, Kim H Andreassen, Susanne S Osther, Helene U Jung. Pathophysiological aspects of ureterorenoscopic management of upper urinary tract calculi. Curr Opin Urol. 2016;26:63-9.

6. Wang Y, Zhong B, Yang X, et al. Comparison of the efficacy and safety of URSL, RPLU, and MPCNL for treatment of large upper impacted ureteral stones: a randomized controlled trial. BMC Urol 17:17.

7. Preminger GM, Tiselius HG, Assimos DG, et al. Guideline for the management of ureteral calculi. J Urol 2007;178:2418-2434.

8. Eric L,Martin L,Olivier $\mathrm{N}$,et al. Transurethral ureterorenolithotripsy using new automated irrigation/suction system controlling pressure and flow compared with standard irrigation: A randomized pilot study. Journal of endourology, 2003,17: 97-101.

9. Philipp Gild, Luis A Kluth, Malte W Vetterlein, Oliver Engel, Felix K H Chun, Margit Fisch. Adult iatrogenic ureteral injury and stricture-incidence and treatment strategies. Asian J Urol. 2018;5:101106.

10. Du C, Song L, Wu X, Deng X, Yang Z, Zhu X, Zhu L, He J. A study on the clinical application of a patented perfusion and suctioning platform and ureteral access sheath in the treatment of large ureteral stones below L4 level. Int Urol Nephrol. 2019;51:207-213.

11. David C,Timothy D.Holmium laser percutaneous nephrolithotomy using a unique suction device.Journal of endourology, 2004;18:780-782.

12. Zewu Zhu,Yu Cui,Feng Zeng,et al.Comparison of suctioning and traditional ureteral access sheath during flexible ureteroscopy in the treatment of renal stones. World Journal of Urology.2019,37:921929.

13. Deng X, Song L, Xie D, et al. A Novel Flexible ureteroscopy with intelligent control of renal pelvic pressure: an initial experience of 93 cases. J Endourol 2016,30:1067-1072 
14. Jad Khaled AlSmadi,Xiaohang Li, Guohua Zeng, et al. Use of a modifified ureteral access sheath in semi-rigid ureteroscopy to treat large upper ureteral stones is associated with high stone free rates. Asian Journal of Urology, 2019,6:217-221.

15. Kaplan AG, Lipkin ME, Scales Jr CD, et al. Use of ureteral access sheaths in ureteroscopy. Nat Rev Urol 2016;13: 135-140.

16. Kourambas J, Byrne RR, Preminger GM. Does a ureteral access sheath facilitate ureteroscopy? J Urol 2005;165:789-793.

17. Deng X, Song L, Xie D, Fan D, Zhu L, Yao L, Wang X, Liu S, Zhang Y, Liao X, Liu S, Peng Z, Hu M, Zhu X, Huang J, Liu T, Du C, Guo S, Yang Z, Peng G, Ye Z.A Novel Flexible Ureteroscopy with Intelligent Control of Renal Pelvic Pressure: An Initial Experience of 93 Cases. J Endourol. 2016;30:1067-1072

18. Sung Yong Cho, Seung Hoon Ryang, Dong Sup Lee. A presumptive role of lower ureteral angles in the difficulty of ureteral access sheath insertion during retrograde intrarenal surgery. Int Urol Nephrol 2020;52:1657-1663.

19. Chuance Du,Leming Song,Xiaoyuan Wu,et al.A study on the clinical application of a patented perfusion and suctioning platform and ureteral access sheath in the treatment of large ureteral stones below L4 level.International Urology and Nephrology ,2019,51:207-213.

20. Tokas T, Skolarikos A, Herrmann TRW, Nagele U; Training and Research in Urological Surgery and Technology (T.R.U.S.T.)-Group.Pressure matters 2: intrarenal pressure ranges during upper-tract endourological procedures. World J Urol. 2019;37:133-142.

21. Fan S,Gong B,Hao Z,et a1.Risk factors of infectious complications following flexible ureteroscope with a holmium laser:a retrospective study.Int J Clin Exp Med,2015;8:11252-11259.

22. Deng $X$,Song $L$,Xie $D$, et a1.Predicting outcomes after minimally percutaneous nephrolithotomy with the aid of a patented system by using the Guy'S stone score.Urol Int,2016,97:67-71.

\section{Figures}


Figure 1.1

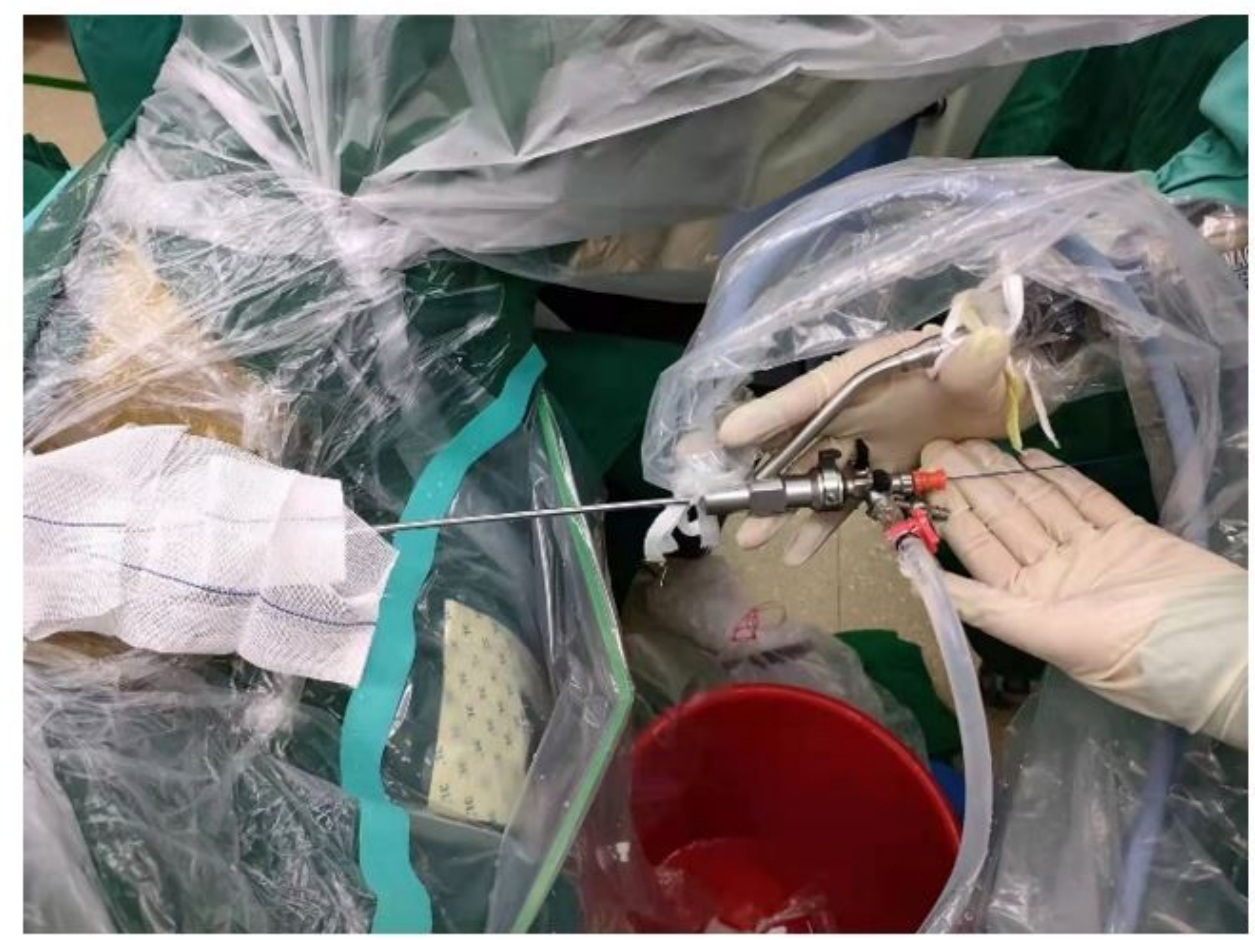

Figure 1.2

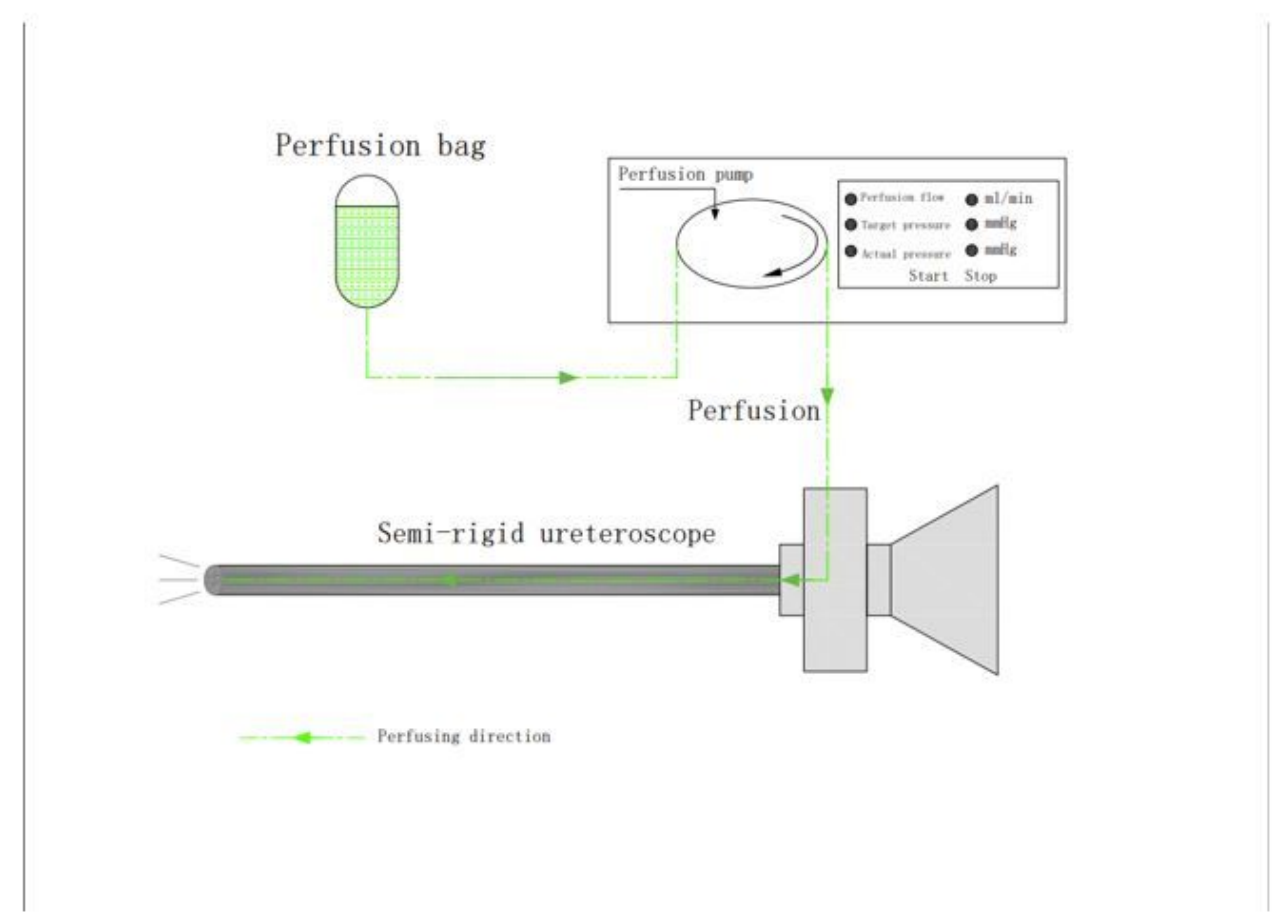

Figure 1

1.1 Traditional semi-rigid ureteroscopic lithotripsy

1.2 Sketch map of traditional semi-rigid ureteroscopic lithotripsy 
Figure 2.1

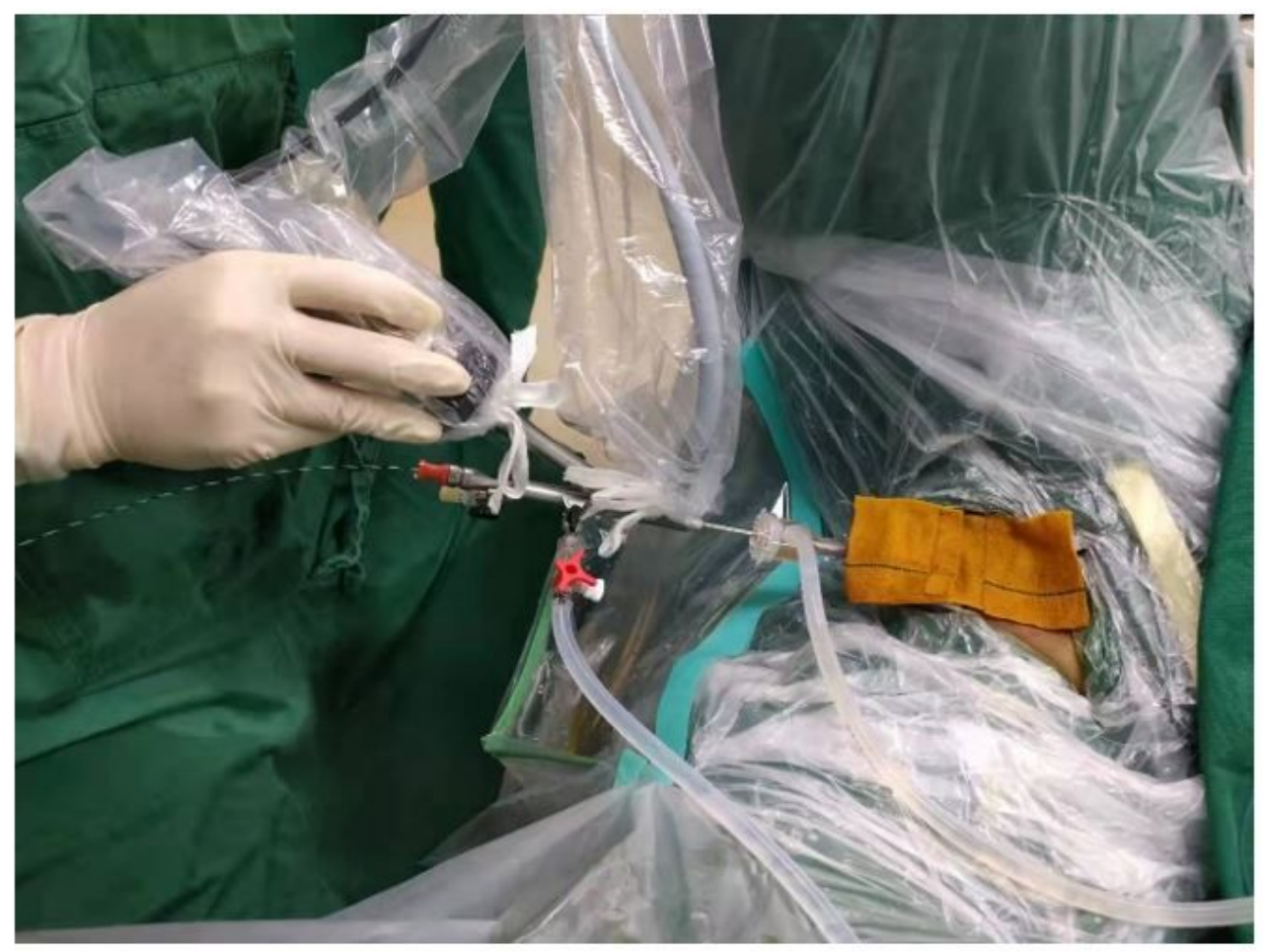

Figure 2.2
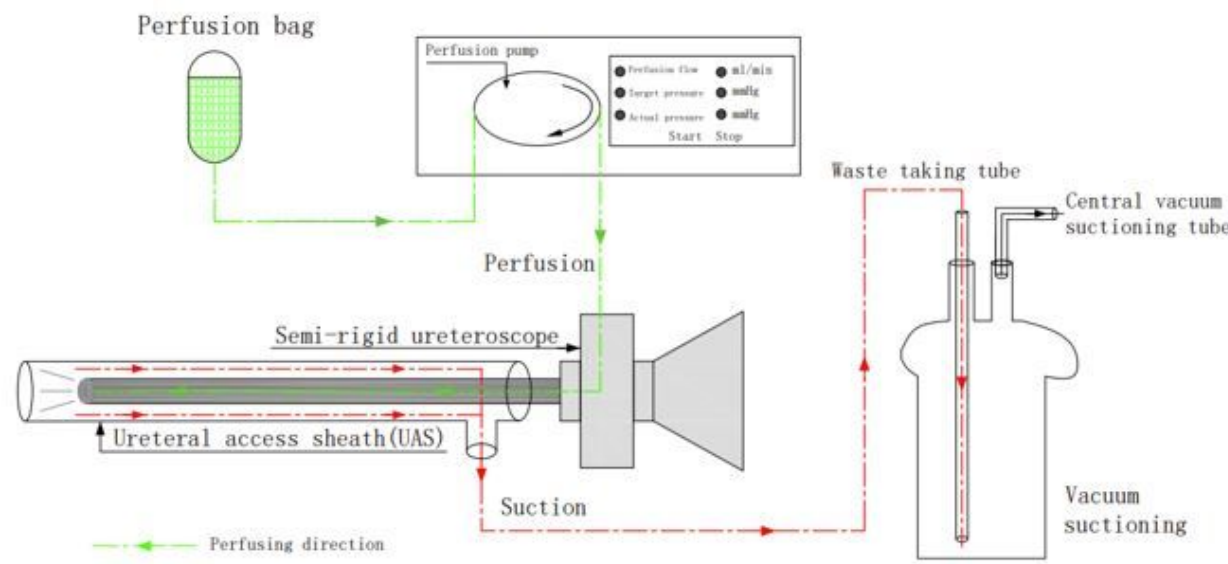

Figure 2

2.1 Suctioning semi-rigid ureteroscopic lithotripsy with a sheath

2.2 Sketch map of suctioning semi-rigid ureteroscopic lithotripsy with a sheath 
Figure 3.1

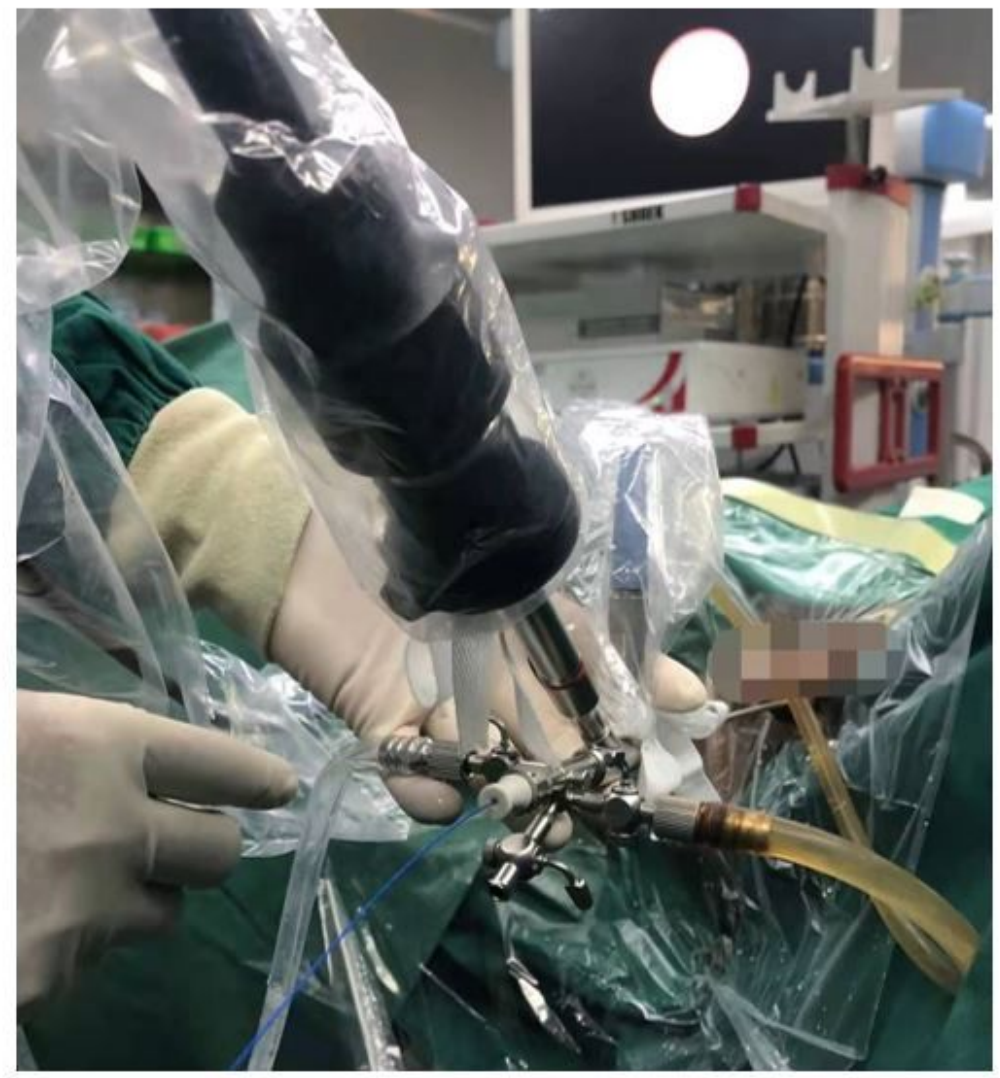

Figure 3.2

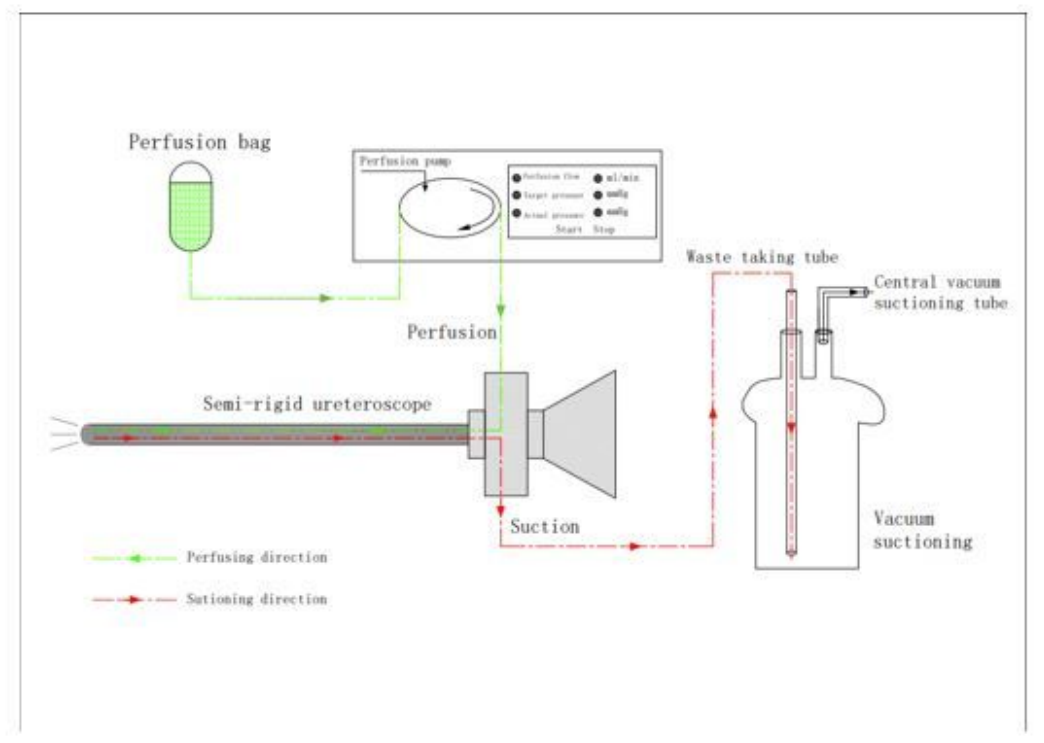

Figure 3

3.1 A new type of suctioning integrated rigid ureteroscopic lithotripsy

3.2 Sketch map of a new type of suctioning integrated rigid ureteroscopic lithotripsy

\section{Supplementary Files}


This is a list of supplementary files associated with this preprint. Click to download.

- PatientsData.xIsx 